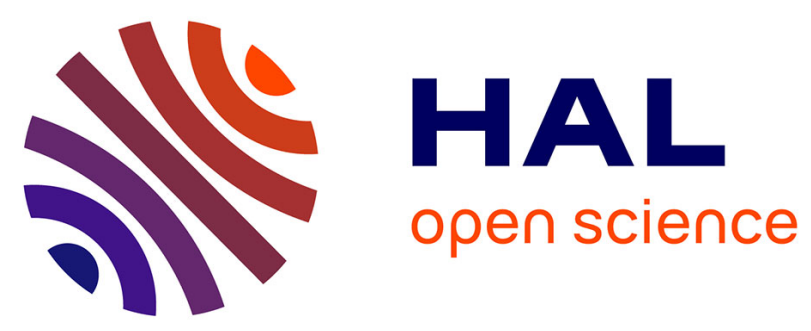

\title{
Topology Optimization of Piezoelectric Plate Energy Harvester Under External In-Plan Force Considering Different Boundary Conditions
}

\author{
Abbas Homayouni, Abdenbi Mohand Ousaid, Micky Rakotondrabe
}

\section{- To cite this version:}

Abbas Homayouni, Abdenbi Mohand Ousaid, Micky Rakotondrabe. Topology Optimization of Piezoelectric Plate Energy Harvester Under External In-Plan Force Considering Different Boundary Conditions. International Conference on Manipulation, Automation and Robotics at Small Scales, Jul 2019, Helsinki, Finland. 10.1109/MARSS.2019.8860967 . hal-02399242

HAL Id: hal-02399242

https://hal.science/hal-02399242

Submitted on 9 Dec 2019

HAL is a multi-disciplinary open access archive for the deposit and dissemination of scientific research documents, whether they are published or not. The documents may come from teaching and research institutions in France or abroad, or from public or private research centers.
L'archive ouverte pluridisciplinaire HAL, est destinée au dépôt et à la diffusion de documents scientifiques de niveau recherche, publiés ou non, émanant des établissements d'enseignement et de recherche français ou étrangers, des laboratoires publics ou privés. 


\title{
Topology Optimization of Piezoelectric Plate Energy Harvester Under External In-Plan Force Considering Different Boundary Conditions
}

\author{
Abbas Homayouni Amlashi ${ }^{1}$, Abdenbi Mohand-Ousaid ${ }^{1}$ and Micky Rakotondrabe ${ }^{1}$
}

\begin{abstract}
In this paper, the goal is to optimize the electrical power of a rectangular piezoelectric plate which is under application of external in-plan force. The most challenging problem for in-plane force is the charge cancellation which is due to combination of tension and compression in different parts of the plate resulted by the external force. To remedy, the topology optimization method is utilized to find the best possible design of the piezoelectric plate to overcome the charge cancellation and produce maximum voltage. To do so, first a detailed two dimensional finite element modelling of the piezoelectric material suitable for topology optimization is presented. Then, the topology optimization method known as solid isotropic material with penalization (SIMP) is extended for the 2D FEM piezoelectric model which is faster than the 3D FEM modelling in case of in-plan force and stress. The performance of the topology optimization is investigated for different boundary conditions in addition to classical cantilever plate with clamped-free boundary condition. Finally, the optimized designs for different boundary conditions are implemented in COMSOL multiphysics platform. The results demonstrate that the optimized design by topology optimization method can produce more voltage in comparison to simple full plate while having less volume of piezoelectric material.
\end{abstract}

\section{INTRODUCTION}

In recent years, a huge industrial and research budget is devoted to smart materials specially the piezoelectric materials. Thanks to their electromechanical coupling effect, they have applications in three main areas: actuation using inverse piezoelectric effect, sensing and energy harvesting using the direct piezoelectric effect. Due to their satisfying power density at small scales [1] they have applications in micro-electromechanical systems (MEMS) [2] and wireless sensor networks (WSN) [3] for the purpose of sensing or energy harvesting. In these applications, for non piezoelectric sensors, energy refueling is a critical challenge since sometimes there is no access to sensors for changing the batteries. Even solar panels are not a perfect solution in places where there is no sunlight. Besides solar panels needs a considerable surface to collect the energy from light sources which is not possible in small scale applications. That's why the researchers considered the piezoelectric materials as an alternative solution which can convert the mechanical energy of the environment to the electrical energy. On the other

*This work has been supported by the national CODE-TRACK project (ANR-17-CE05-0014-01, Control theory tools for optimal design of piezoelectric energy harvesters devoted to birds tracking devices). This work has also been partially supported by the Bourgogne France-Comté region project COMPACT.

${ }^{1}$ Authors are with the Department Automatique et Systèmes MicroMécatroniques, FEMTO-ST Institute, Université Bourgogne FrancheComté, CNRS, Besançon 25000, France. abbas.homayouni@femtost.fr;abdenbi.mohand@femto-st. fr;mrakoton@femto-st.fr hand, although piezoelectric materials provide acceptable power densities in small scales, researchers tried to optimize their power densities in many ways including parametric optimization of the piezoelectric structures [4], [5], increasing the bandwidth [6], designing their structures on the basis of interval techniques [7] such that prescribed performances are robustly satisfied [8], designing nonlinear and Bi-stable systems [9], optimization of the electrical circuit [10], etc.

One of the recent approaches for optimization of the piezoelectric energy harvesters is using the topology optimization (TO) method [11]. The basic idea behind this method, is to distribute the material in a design domain to minimize a cost function subjected to one or several constraints while using finite element model of the system. The works in the area of TO primarily focused on compliance problems in which the goal was minimizing the structure's deformation in a predefined boundary and load conditions. Among many works in this area, Sigmund [12] proposed a 99 line code which implements the TO for the compliance problem and it shows a promising performance to reach the optimal designs. This code later used as main engine for developing other codes in the area of compliance problems [13], [14]. The idea behind the TO is also interesting for the researchers to optimize the power density of the piezoelectric structures. To do so, they defined different cost functions and constraints to optimize the power density of the piezoelectric energy harvesters under static force [15], dynamic or harmonic force [16] and random force [17]. However, in all of the aforementioned researches, the main configuration of the piezoelectric structure is cantilever plate in which the boundary condition is a classical clamped-free boundary condition. This configuration is interesting for the researchers since it is easy for real implementation. Therefore, to the knowledge of the authors, there is no work in the area of using TO for piezoelectric energy harvesters considering other boundary conditions as alternatives for classical clamped-free beam.

Generally, in case of in-plane force for the piezoelectric material, stack configuration has been used to take advantage of bigger $d_{33}$ piezoelectric electromechanical coefficient in comparison to $d_{31}$ [3] which results bigger electrical output due to mechanical input. On the other hand, piezoelectric stack configuration has bigger stiffness in direction of the polarization which prevents big deformation and decreases the efficiency of energy harvesting. In addition, stack configuration can just absorb energy from the force in direction of the polarization. However, a plate with polarization direction perpendicular to the plan of plate can absorb energy from forces in plane of plate in 2 different directions. On the 
other hand, the problem of in plan forces for plates is that it induces tension on some part of the plate and compression on other parts of the plate. This results in opposite sign of local potentials on the surface of same electrode which known as charge cancellation and it will reduce the final harvested electrical energy.

In this paper, we suggest using the piezoelectric plate energy harvester to harvest the energy from in-plane external force and taking advantage of lower stiffness of plate in comparison to stack configuration. Different boundary conditions are imposed on the plate to investigate the energy harvesting performance for each of them. To tackle the charge cancellation problem and maximizing the harvested energy topology optimization is utilized to modify the structure of the plate based on the direction of the applied force and boundary conditions.

The structure of the paper is as follows: in section two, a detailed two dimensional finite element modelling for piezoelectric plate is presented. The FEM is presented in a way which makes it easy for implementation in TO. In section three, the cost function proposed by Zheng [15] is used to maximize the electrical energy for the given input mechanical energy so the final optimized results can be used for energy harvesting purpose or sensor applications. The sensitivity of cost function regarding each element's density is calculated by a method known as Solid Isotropic Material with Penalization (SIMP) which considers intermediary densities for the elements. Section four is devoted to simulation results in which a rectangular design domain is considered to harvest the electrical energy from a given amplitude of input force. The optimized results by TO are transferred to COMSOL multiphysics platform and the power density and terminal charge of the electrode for different configurations are compared to each other.

\section{Modelling}

\section{A. 2D Finite Element Modelling of Piezoelectric Material}

The linear coupled mechanical and electrical behavior of piezoelectric materials by neglecting the thermal coupling can be written in the following compact matrix form [15], [16],

$$
\begin{gathered}
T=c^{E} S-e E \\
D=e^{T} S+\varepsilon^{S} E
\end{gathered}
$$

In which [18],

$$
\begin{gathered}
c=\left[\begin{array}{ccc}
c_{11} & c_{13} & 0 \\
c_{13} & c_{33} & 0 \\
0 & 0 & c_{55}
\end{array}\right] \\
e=\left[\begin{array}{ccc}
0 & 0 & e_{15} \\
e_{31} & e_{33} & 0
\end{array}\right] \\
\varepsilon=\left[\begin{array}{cc}
\varepsilon_{11} & 0 \\
0 & \varepsilon_{33}
\end{array}\right]
\end{gathered}
$$

In (1)-(4), $T$ and $S$ are the vectors of mechanical stress and strain while $c^{E}$ is the stiffness matrix. $D$ and $E$ are the vectors of electrical displacement and electrical field. $e$ is the coupling matrix while $T$ shows the transpose of the matrix and $\varepsilon^{S}$ is the matrix of permittivity. To discrete the design domain, the 4 node rectangular element which is the mapped form of bilinear quadrilateral element is employed as shown in Fig. 1. Therefor, for this 4 node element, the displacements in natural coordinates $\xi$ and $\eta$ can be written with the help of the shape functions as follows [19],

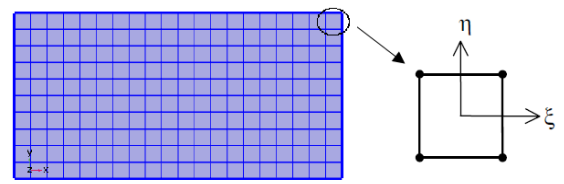

Fig. 1. Plate meshed with bilinear quadrilateral element

$$
\begin{gathered}
\xi=\xi_{1} N_{1}+\xi_{2} N_{2}+\xi_{3} N_{3}+\xi_{4} N_{4} \\
\eta=\eta_{1} N_{1}+\eta_{2} N_{2}+\eta_{3} N_{3}+\eta_{4} N_{4}
\end{gathered}
$$

In which the shape functions are,

$$
\begin{aligned}
& N_{1}=\frac{1}{4}(1-\xi)(1-\eta), N_{2}=\frac{1}{4}(1+\xi)(1-\eta) \\
& N_{3}=\frac{1}{4}(1+\xi)(1+\eta), N_{4}=\frac{1}{4}(1-\xi)(1+\eta)
\end{aligned}
$$

Since the selected element is rectangular itself, no extra mapping is required to transfer the global coordinates $x$ and $y$ to the natural coordinates $\xi$ and $\eta$. Now, by considering additional potential degree of freedom for each node, strain and electric field can be written based on the shape functions in the following form,

$$
\begin{aligned}
& S=B_{u} u \\
& E=B_{\phi} \phi
\end{aligned}
$$

$u$ and $\phi$ are the mechanical displacement and electric potential vector. $B_{u}$ and $B_{\phi}$ are the strain displacement matrices where,

$B_{u}=\left[\begin{array}{cccccccc}\frac{\partial N_{1}}{\partial \xi} & 0 & \frac{\partial N_{2}}{\partial \xi} & 0 & \frac{\partial N_{3}}{\partial \xi} & 0 & \frac{\partial N_{4}}{\partial \xi} & 0 \\ 0 & \frac{\partial N_{1}}{\partial \eta} & 0 & \frac{\partial N_{2}}{\partial \eta} & 0 & \frac{\partial N_{3}}{\partial \eta} & 0 & \frac{\partial N_{4}}{\partial \eta} \\ \frac{\partial N_{1}}{\partial \eta} & \frac{\partial N_{1}}{\partial \xi} & \frac{\partial N_{2}}{\partial \eta} & \frac{\partial N_{2}}{\partial \xi} & \frac{\partial N_{3}}{\partial \eta} & \frac{\partial N_{3}}{\partial \xi} & \frac{\partial N_{4}}{\partial \eta} & \frac{\partial N_{4}}{\partial \xi}\end{array}\right]$

$$
B_{\phi}=\left[\begin{array}{llll}
\frac{\partial N_{1}}{\partial \xi} & \frac{\partial N_{2}}{\partial \xi} & \frac{\partial N_{3}}{\partial \xi} & \frac{\partial N_{4}}{\partial \xi} \\
\frac{\partial N_{1}}{\partial \eta} & \frac{\partial N_{2}}{\partial \eta} & \frac{\partial N_{3}}{\partial \eta} & \frac{\partial N_{4}}{\partial \eta}
\end{array}\right]
$$

Now, by substituting (5)-(9) to (1), after performing a mathematical procedure which is explained in [15], the element matrix equation for deriving the mechanical displacement and electric potential due to external mechanical force $(f)$ and electric charge $(q)$ is [15],

$$
\left[\begin{array}{cc}
k_{u u} & k_{u \phi} \\
k_{\phi u} & -k_{\phi \phi}
\end{array}\right]\left[\begin{array}{l}
u \\
\phi
\end{array}\right]=\left[\begin{array}{l}
f \\
q
\end{array}\right]
$$

In which,

$$
\begin{aligned}
k_{u u} & =\int_{v} B_{u}^{T} c^{E} B_{u} d v, k_{u \phi}=\int_{v} B_{u}^{T} e B_{\phi} d v \\
k_{\phi u} & =\int_{v} B_{\phi}^{T} e B_{u} d v, k_{\phi \phi}=\int_{v} B_{\phi}^{T} \varepsilon B_{\phi} d v
\end{aligned}
$$


In $11, k_{u u}$ is the elastic stiffness matrix, $k_{u \phi}$ and $k_{\phi u}$ are the piezoelectric stiffness matrices and $k_{\phi \phi}$ is the dielectric stiffness matrix. Now, with the following equations, element matrices are assembled to form the global stiffness matrices,

$$
\begin{aligned}
K_{u u} & =\sum_{i=1}^{N E} k_{u u}, K_{u \phi}=\sum_{i=1}^{N E} k_{u \phi} \\
K_{\phi u} & =\sum_{i=1}^{N E} k_{\phi u}, K_{\phi \phi}=\sum_{i=1}^{N E} k_{\phi \phi}
\end{aligned}
$$

Therefore, the global matrix equation is,

$$
\left[\begin{array}{cc}
K_{u u} & K_{u \phi} \\
K_{\phi u} & -K_{\phi \phi}
\end{array}\right]\left[\begin{array}{l}
U \\
\Phi
\end{array}\right]=\left[\begin{array}{l}
F \\
Q
\end{array}\right]
$$

\section{B. Boundary Conditions}

The global matrix equation in (13), can not be solved in this format. Since the stiffness matrices are all singular. To solve this equation, proper mechanical and electrical boundary conditions should be applied on the system. for the electrical boundary condition, the potentials on all edges are considered to be zero which is the case when we have the polling direction on the perpendicular axis to the plate and the electrodes on top and bottom surface of the piezoelectric plate. In this form, the charges on the side surfaces of plate will be zero which in $2 \mathrm{D}$ results in zero charges on all edges.

For the mechanical deformations, classical boundary conditions like clamped, pin and free boundary conditions are considered. By applying the mechanical and electrical boundary conditions on the stiffness matrices, they will be non-singular and then the global matrix equation in (13) can be solved.

\section{TOPOLOGY OPTIMIZATION}

\section{A. Cost Function}

In energy harvesting or sensing applications there is no external electric field applying on the system then $Q=0$. In this case, the goal is to maximize the electrical output of the system regarding the input mechanical force applying on the system. To do so, the mechanical and electrical energy stored in the piezoelectric material are respectively defined in the following form,

$$
\begin{aligned}
& \Pi^{S}=\left(\frac{1}{2}\right) U^{T} K_{u u} U \\
& \Pi^{E}=\left(\frac{1}{2}\right) \Phi^{T} K_{\phi \phi} \Phi
\end{aligned}
$$

Now, a cost function is defined as,

$$
J=\frac{W^{F}}{\Pi^{E}}
$$

By defining the cost function as (16), we pursue to maximize the electrical energy regarding input mechanical force. The work due to the input force can be calculated with the help of (13) as,

$$
\begin{gathered}
W^{F}=\frac{1}{2} U^{T} F=\frac{1}{2}\left(U^{T} K_{u u} U+U^{T} K_{u \phi} \Phi\right)= \\
\frac{1}{2}\left(U^{T} K_{u u} U+\Phi^{T} K_{\phi \phi} \Phi\right)=\Pi^{S}+\Pi^{E}
\end{gathered}
$$

Therefore, the new format of the cost function is,

$$
J=1+\frac{\Pi^{S}}{\Pi^{E}}
$$

To minimize the cost function mentioned in (18), there is a constraint on the volume for the final designed space which can be expressed as,

$$
V(x)=\sum_{i=1}^{N E} x_{i} v_{i} \leq V
$$

where $x_{i}$ is the density of the element $i$ and $N E$ is the maximum number of elements.

\section{B. Solid Isotropic Material with Penalization (SIMP)}

In order to deal with the optimization method mentioned in (18) and (19), there are several topology optimization method like binary compliance problem, Homogenization method and SIMP. These later one is the most famous one which is proposed in detail in several references [11], [12], [18] for compliance problems. The first step in implementation of SIMP method is to do the sensitivity analysis in which the sensitivity of cost function regarding each element is derived in the following format [15],

$$
\begin{gathered}
\frac{\partial J}{\partial x_{i}}=\frac{1}{\Pi_{i}^{E}}\left[\left(\frac{1}{2} u_{i}^{T}+\lambda_{1, i}^{T}\right) \frac{\partial k_{u u, i}}{\partial x_{i}} u_{i}+\lambda_{1}^{T} \frac{\partial k_{u \phi, i}}{\partial x_{i}} \phi_{i}\right. \\
\left.+\mu_{1, i}^{T} \frac{\partial k_{\phi u, i}}{\partial x_{i}} u_{i}-\mu_{1, i}^{T} \frac{\partial k_{\phi \phi, i}}{\partial x_{i}} \phi_{i}\right]-\frac{\Pi_{i}^{S}}{\left(\Pi_{i}^{E}\right)^{2}}\left[\frac{1}{2} \phi_{i}^{T} \frac{\partial k_{\phi u, i}}{\partial x_{i}} \phi_{i}-\right. \\
\left.\mu_{2, i}^{T} \frac{\partial k_{\phi \phi, i}}{\partial x_{i}} \phi_{i}+\lambda_{2, i}^{T} \frac{\partial k_{u u, i}}{\partial x_{i}} u_{i}+\lambda_{2}^{T} \frac{\partial k_{u \phi, i}}{\partial x_{i}} \phi_{i}+\mu_{2}^{T} \frac{\partial k_{\phi u, i}}{\partial x_{i}} u_{i}\right]
\end{gathered}
$$

It should be noted that the sensitivity analysis is performed on the element matrices in which $\lambda$ and $\mu$ are the element size adjoint vectors which can be calculated by the following global matrix equation,

$$
\begin{aligned}
& {\left[\begin{array}{cc}
K_{u u} & K_{u \phi} \\
K_{\phi u} & -K_{\phi \phi}
\end{array}\right]\left[\begin{array}{l}
\Lambda_{1} \\
\Upsilon_{1}
\end{array}\right]=\left[\begin{array}{c}
-K_{u u} U \\
0
\end{array}\right]} \\
& {\left[\begin{array}{cc}
K_{u u} & K_{u \phi} \\
K_{\phi u} & -K_{\phi \phi}
\end{array}\right]\left[\begin{array}{l}
\Lambda_{2} \\
\Upsilon_{2}
\end{array}\right]=\left[\begin{array}{c}
0 \\
-K_{\phi \phi} \Phi
\end{array}\right]}
\end{aligned}
$$

In (21), $\Lambda$ and $\Upsilon$ are the global adjoint vectors which should be resolved to give the element adjoint vectors $\lambda$ and $\mu$ respectively. But the other problem in sensitivity equation in (20) is the derivative of stiffness matrices respect to element densities $\left(\frac{\partial k_{x x, i}}{\partial x_{i}}\right)$ which leads us to the core conception of the SIMP methodology that is based on the material density distribution. In this methodology, density of each element can have a continuous values between 0 and 1 . Therefore, in the color space of the design domain, if one considers white for the zero density and black for the density equal to one, then in the SIMP method we can have also grey elements which have densities between 0 and 1 . However, the problem with grey elements is the production of the final design for the real applications. Therefore, a penalization factor $p$ is defined to push the optimized design toward the 0 and 1 structure. Based on the aforementioned explanation, 
SIMP method started by defining a relation between element density and element's young's modulus of elasticity [11],

$$
\bar{E}_{i}=\bar{E}_{i}\left(x_{i}\right)=x_{i}^{p} \bar{E}_{0}, x_{i} \in[0,1]
$$

In (22), $\bar{E}_{0}$ is the young's modulus of elasticity of the isotropic material. For non-isotropic piezoelectric materials the same concept as (22) applied for all of the stiffness matrices,

$$
k_{u u, i}(x)=x_{i}^{p} k_{u u}^{e}, k_{u \phi, i}(x)=x_{i}^{p} k_{u \phi}^{e}, k_{\phi \phi, i}(x)=x_{i}^{p} k_{\phi \phi}^{e}
$$

Still some numerical instabilities like mesh dependencies, local minima, etc. can be encountered. To deal with such kinds of problems density filter should be used which is explained in 99 line MATLAB code by Sigmund [12] and re-mentioning those explanations is avoided here. After applying the density filter, following equations can be written for the derivatives of the stiffness matrices[16],

$$
\begin{aligned}
& \frac{\partial k_{u u, i}(\tilde{x})}{\partial \tilde{x}_{i}}=p \tilde{x}^{p-1} k_{u u} \\
& \frac{\partial k_{u \phi, i}(\tilde{x})}{\partial \tilde{x}_{i}}=p{\tilde{x_{i}}}^{p-1} k_{u \phi} \\
& \frac{\partial k_{\phi \phi, i}(\tilde{x})}{\partial \tilde{x}_{i}}=p \tilde{x}^{p-1} k_{\phi \phi}
\end{aligned}
$$

In which, $\tilde{x}$ is the density of the element after applying the density filter. It should be noted that $p$ can be different for each stiffness matrix. However, here it is considered to be equal for all of them. After sensitivity analyis for each element, to optimize the densities for all elements based on the (18) respect to the volume constraint in (19), Optimality

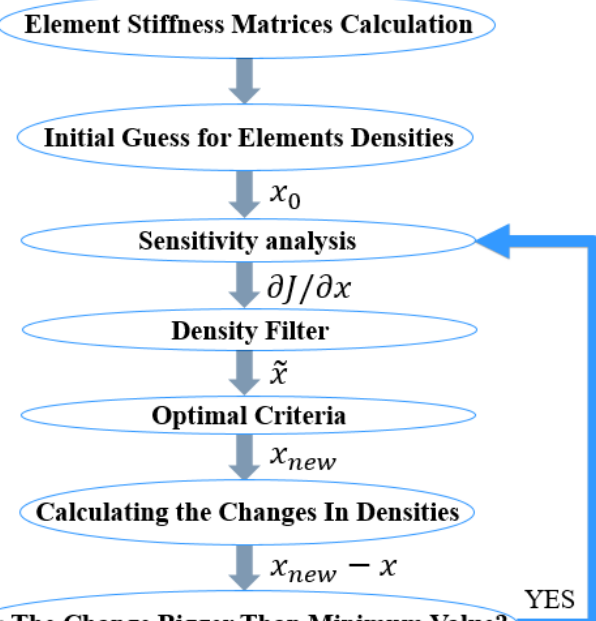

Is The Change Bigger Than Minimum Value?

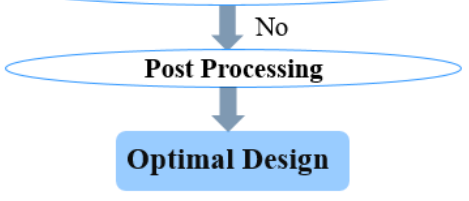

Fig. 2. Topology Optimization Algorithm
Criteria (OC) method is used. This method is an iterative method to update the design variables for minimizing the cost function based on the constraint. The MATLAB implementation algorithm for this method also mentioned in [12] and the same line of codes of that paper is used in the algorithm of this paper.

The general diagram of topology optimization method which is described above can be seen in Fig. 2. The general optimality criteria method stops when there are no significant changes in design variables (here are densities). In this case it is necessary to define a minimum value of changes in densities. For compliance problem mentioned in [12], the minimum value is considered to be 0.01 . In compliance problems, the cost function just consists of mechanical work due to external force. However, the cost function in this paper is a fraction of mechanical work to the electrical output. Then, changing the densities for minimization of mechanical deformation may lead to minimization of the electrical output and vice versa. Therefore, the minimum change of densities should be considered bigger than the compliance problems or a maximum number of iteration should be defined for the code. In the former stopping method it is possible to be trapped in the local optimums.

When the optimization stops, the next step is post processing. Actually, it is true that penalization will push the densities to zero and one. However, still in the final design, there are some grey elements. To tackle this problem, the post processing method mentioned in [20] which consist two steps of Gaussian filter and thresholding is used here.

\section{RESUlTS}

In this section, the results of TO algorithm on the design of effective (EH or sensor) for different configuration is presented. A rectangular design domain with $10 \mathrm{~mm} \times 5 \mathrm{~mm}$ with $0.2 \mathrm{~mm}$ thickness is chosen and it is meshed by $200 \times$ 100 rectangular element. The piezoelectric material for this plate is (PZT-4) with the polarization direction perpendicular to the plate (in $\mathrm{z}$ direction) and full characteristics are mentioned in table I. A point force with amplitude of 1 newton or two point force with amplitude of 0.5 newton are considered as external mechanical force. To analyze the performance of the optimized design, COMSOL multiphysics is used to evaluate the voltage and charge due to applied force on the optimized design and the result is also compared with the full plate performance. the applied force is considered to be static and harmonic with frequency of $1 \mathrm{KHZ}$.

TABLE I

PIEZOELECTRIC PLATE PROPERTIES

\begin{tabular}{|c|c|c|c|}
\hline Parameter & Value & Parameter & Value \\
\hline \hline$c_{11}$ & $1.38999 \mathrm{e}+011[\mathrm{~Pa}]$ & $e_{15}$ & $12.7179\left[\mathrm{C} / \mathrm{m}^{2}\right]$ \\
\hline$c_{13}$ & $7.42836 \mathrm{e}+010[\mathrm{~Pa}]$ & $\varepsilon_{11}$ & $762.5 \times \varepsilon_{0}$ \\
\hline$c_{33}$ & $1.15412 \mathrm{e}+011[\mathrm{~Pa}]$ & $\varepsilon_{33}$ & $663.2 \times \varepsilon_{0}$ \\
\hline$c_{55}$ & $2.5641 \mathrm{e}+010[\mathrm{~Pa}]$ & Length & $10[\mathrm{~mm}]$ \\
\hline$e_{31}$ & $-5.20279\left[\mathrm{C} / \mathrm{m}^{2}\right]$ & Width & $5[\mathrm{~mm}]$ \\
\hline$e_{33}$ & $15.0804\left[\mathrm{C} / \mathrm{m}^{2}\right]$ & Thickness & $2[\mathrm{~mm}]$ \\
\hline
\end{tabular}



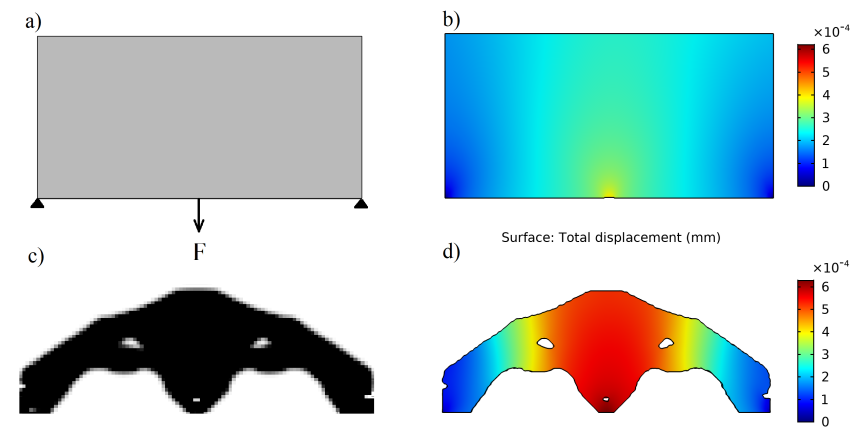

Fig. 3. Configuration: 1 force PP, a) Design domain with boundary conditions and applied force, b) Full plate deformation, c) Topology optimization result, d) Deformation of the optimized design

In figures 3 to 7 different optimized design for different boundary conditions and different application force are shown. In the first part of these figures the design domain with defined boundary conditions and point of the applied force is illustrated. In the second part of these figures the deformation of the full plate due to applied force are shown. In part c, the proposed design by TO is illustrated then the deformation of this optimized design due to applied force is shown in the last part of the figures.

It is important to note that in all of the figures, the distribution of the deformation in the optimized design is much more than the full plate deformation. The reason is that the full plate is much more stiffer than the optimized design while in piezoelectric material more strain is required to produce more electrical charge. Therefore, this better distribution of displacement results in more applied voltage in piezoelectric material which are reported in table II.

In Fig. 3, force is applied on the plate which is rested on two pins. The pin element gives the rotation freedom to the elements in the vicinity of the pins. The TO proposed a design in which applied force results in compressive stress in the most part of the design while in full plate the stress can be tension near to the force application point and compressive stress in upper part of the plate. This is highly important since a combination of compression and tension in electrodes
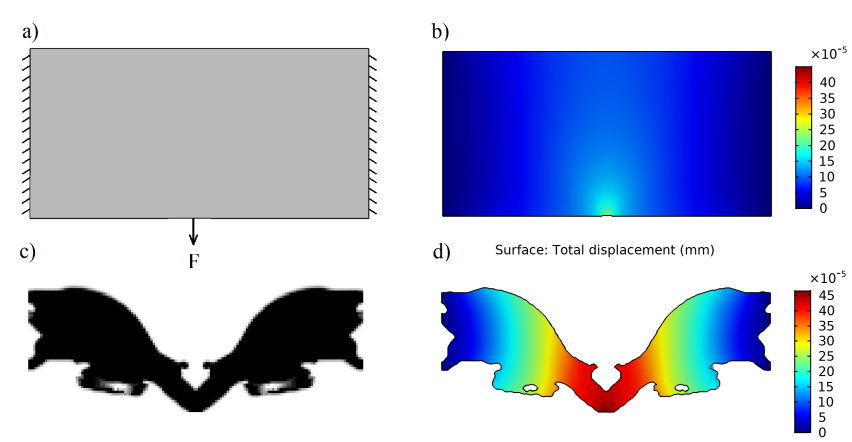

Fig. 4. Configuration: 1 force $\mathrm{CC}$, a) Design domain with boundary conditions and applied force, b) Full plate deformation, c) Topology optimization result, d) Deformation of the optimized design
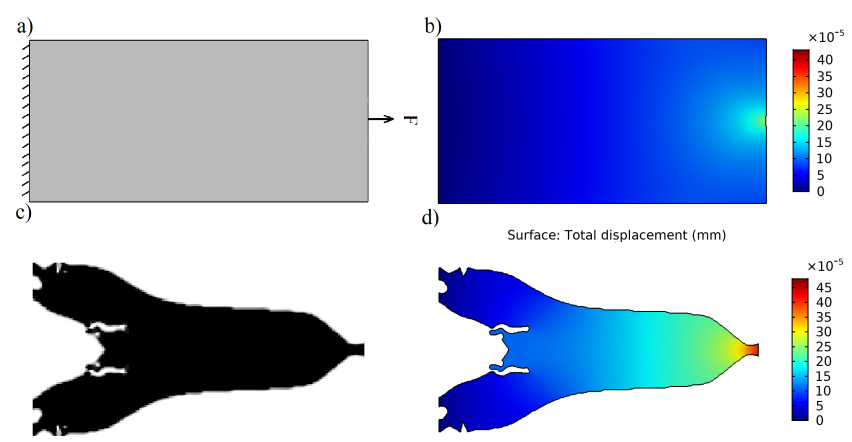

Fig. 5. Configuration: 1 force Longitudinal $\mathrm{CF}$, a) Design domain with boundary conditions and applied force, b) Full plate deformation, c) Topology optimization result, d) Deformation of the optimized design

may lead to cancellation of charges and reducing the final voltage of the piezoelectric material. By referring to table II, it is obvious that the power density of the optimized design is more than 2 times bigger than the power density of the full plate.

In Fig. 4, force is applied on the plate which is clamped on both sides. The clamped boundary condition is more strong than pin boundary condition since it prevents the elements from rotation near to the clamped side. By comparing the total displacement in optimized design and the full plate, it is obvious that the total deformation in optimized design is severely more than the full plate since the clamped boundary condition is a strong boundary condition which prevents the deformations in plate. On the other hand, almost all part of the optimized design are under tension while it is expected to have a combination of tension and compression in the full plate.

By inspecting the table II, it is interesting to see that the power density of the optimized design for 1 force clampedclamped is significantly more than the power density of full plate (almost 6 times bigger). In addition, this power density is even more than the power density of the previous optimized design of the 1 force Pin-Pin condition. It is inferred that the TO algorithm take advantage of stronger clamped boundary condition to provide more voltage in the
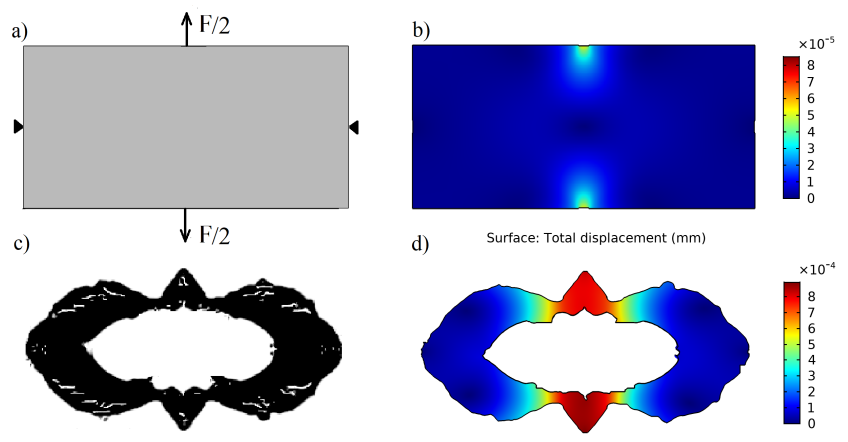

Fig. 6. Configuration: 2 force Longitudinal PP, a) Design domain with boundary conditions and applied force, b) Full plate deformation, c) Topology optimization result, d) Deformation of the optimized design 
TABLE II

ENERGY HARVESTING PERFORMANCE FOR DIFFERENT CONFIGURATION

\begin{tabular}{|c|c|c|c|c|c|c|}
\hline 90 Configuration & Volume & Voltage for $1 \mathrm{KHZ}$ force & Charge for Static Force & Power Density & Full Plate Power Density & Full Plate Voltage \\
\hline 1 Force PP & $5.0261\left[\mathrm{~mm}^{3}\right]$ & $1.52 \mathrm{E}-02[\mathrm{v}]$ & $2.41 \mathrm{E}+00[\mathrm{nC}]$ & $22.83\left[\mathrm{nW} / \mathrm{mm}^{3}\right]$ & $8.51\left[\mathrm{nW} / \mathrm{mm}^{3}\right]$ & $1.30 \mathrm{E}-02[\mathrm{v}]$ \\
\hline 1 Force CC & $5.0702\left[\mathrm{~mm}^{3}\right]$ & $2.31 \mathrm{E}-02[\mathrm{v}]$ & $4.21 \mathrm{E}+00[\mathrm{nC}]$ & $52.43\left[\mathrm{nW} / \mathrm{mm}^{3}\right]$ & $8.52\left[\mathrm{nW} / \mathrm{mm}^{3}\right]$ & $1.31 \mathrm{E}-02[\mathrm{v}]$ \\
\hline 2 Force Latitudinal PP & $5.0117\left[\mathrm{~mm}^{3}\right]$ & $2.42 \mathrm{E}-02[\mathrm{v}]$ & $3.85 \mathrm{E}+00[\mathrm{nC}]$ & $58.6\left[\mathrm{nW} / \mathrm{mm}^{3}\right]$ & $7.48\left[\mathrm{nW} / \mathrm{mm}^{3}\right]$ & $1.37 \mathrm{E}-02[\mathrm{v}]$ \\
\hline 1 Force Longitudinal CF & $4.9968\left[\mathrm{~mm}^{3}\right]$ & $4.02 \mathrm{E}-02[\mathrm{v}]$ & $6.40 \mathrm{E}+00[\mathrm{nC}]$ & $164.32\left[\mathrm{nW} / \mathrm{mm}^{3}\right]$ & $28.037\left[\mathrm{nW} / \mathrm{mm}^{3}\right]$ & $2.59 \mathrm{E}-02[\mathrm{v}]$ \\
\hline 2 Force Longitudinal PP & $5.0173\left[\mathrm{~mm}^{3}\right]$ & $2.03 \mathrm{E}-02[\mathrm{v}]$ & $3.22 \mathrm{E}+00[\mathrm{nC}]$ & $41\left[\mathrm{nW} / \mathrm{mm}^{3}\right]$ & $12.8\left[\mathrm{nW} / \mathrm{mm}^{3}\right]$ & $1.60 \mathrm{E}-02[\mathrm{v}]$ \\
\hline
\end{tabular}
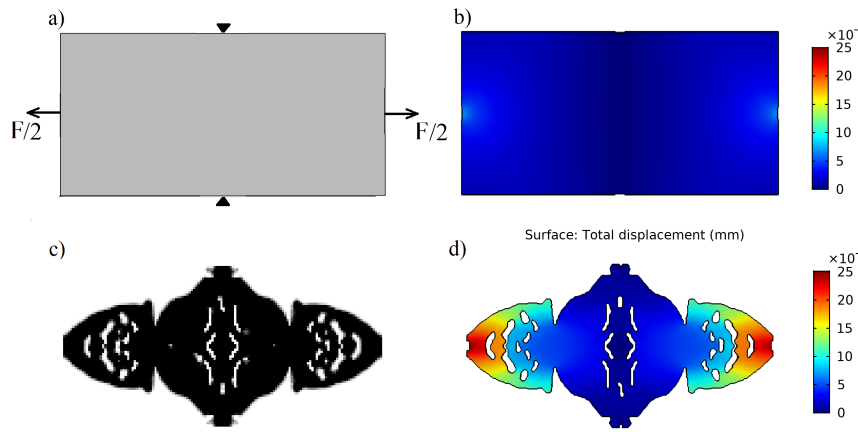

Fig. 7. Configuration: 2 force latitudinal PP a) Design domain with boundary conditions and applied force, b) Full plate deformation, c) Topology optimization result, d) Deformation of the optimized design

piezoelectric material.

In Fig. 5, the classical configuration of clamped-free plate is shown. The total deformation shows a greater deformation for the optimized design. In this configuration all area of the design for full plate and optimized one are under tension. So, this configuration has now charge cancellation problem. By referring to table II it becomes clear that the optimized design has a huge power density.

But Now consider the configuration in Fig. 6 which is a rectangular plate under tension of two force with the amplitude of F/2. So, the over all amplitude of the force remains the same in comparison to previous designs. Again, the optimized design is under tension in most of the design area and it has more deformation in comparison to full plate. By checking the table II, it is clear that this configuration has a power density almost equal to 1 force clamped-clamped configuration. The last configuration can be seen in Fig. 7 in which the rectangular plate is under tension of two force in the longitudinal direction of the plate.

\section{CONCLUSIONS}

In this paper, topology optimization method is used to maximize the harvested electrical power for a rectangular PZT plate under different configurations of force and boundary conditions with finding the best design by considering the static force applied on the design domain. Comparison between the power density of the optimized design and the simple full plate design reveals that topology optimization method was successful to overcome the problem of charge cancellation which is common in plan applied force. Future work would investigate the optimization of the plate to harvest the energy from forces that come from different directions at the same time.

\section{REFERENCES}

[1] H. S. Kim, J.-H. Kim, and J. Kim, "A review of piezoelectric energy harvesting based on vibration," International journal of precision engineering and manufacturing, vol. 12, no. 6, pp. 1129-1141, 2011.

[2] S.-G. Kim, S. Priya, and I. Kanno, "Piezoelectric mems for energy harvesting," MRS bulletin, vol. 37, no. 11, pp. 1039-1050, 2012.

[3] A. Toprak and O. Tigli, "Piezoelectric energy harvesting: State-of-theart and challenges," Applied Physics Reviews, vol. 1(3), 2014.

[4] T. Schlinquer, A. Mohand-Ousaid, and M. Rakotondrabe, "Optimal design of a unimorph piezoelectric cantilever devoted to energy harvesting to supply animal tracking devices," IFAC-PapersOnLine, vol. 50, no. 1, pp. 14600-14605, 2017.

[5] K. Rabenorosoa and M. Rakotondrabe, "Performances analysis of piezoelectric cantilever based energy harvester devoted to mesoscale intra-body robot," in Next-Generation Robotics II; and Machine Intelligence and Bio-inspired Computation: Theory and Applications IX, vol. 9494. International Society for Optics and Photonics, Conference Proceedings, p. 94940E.

[6] D. Zhu, M. J. Tudor, and S. P. Beeby, "Strategies for increasing the operating frequency range of vibration energy harvesters: a review," Measurement Science and Technology, vol. 21, no. 2, p. 022001, 2009.

[7] M. Rakotondrabe, "Performances inclusion for stable interval systems," 2011, pp. 4367-4372.

[8] S. Khadraoui, M. Rakotondrabe, and P. Lutz, "Optimal design of piezoelectric cantilevered actuators with guaranteed performances by using interval techniques," Transactions on Mechatronics, vol. 19, no. 5, pp. 1660-1668, 2014.

[9] R. Harne and K. Wang, "A review of the recent research on vibration energy harvesting via bistable systems," Smart materials and structures, vol. 22, no. 2, p. 023001, 2013.

[10] S. Chamanian, H. Ulusan, A. Koyuncuoglu, A. Muhtaroglu, and H. Kulah, "An adaptable interface circuit with multi-stage energy extraction for low power piezoelectric energy harvesting mems," IEEE Transactions on Power Electronics, 2018.

[11] M. P. Bendsoe and O. Sigmund, Topology optimization, year $=2013$. Springer Science \& Business Media.

[12] O. Sigmund, "A 99 line topology optimization code written in matlab," Structural and multidisciplinary optimization, vol. 21, 2001.

[13] V. J. Challis, "A discrete level-set topology optimization code written in matlab," Structural and multidisciplinary optimization, vol. 41, no. 3, pp. 453-464, 2010.

[14] K. Liu and A. Tovar, "An efficient 3d topology optimization code written in matlab," Structural and Multidisciplinary Optimization, vol. 50, no. 6, pp. 1175-1196, 2014.

[15] B. Zheng, C.-J. Chang, and H. C. Gea, "Topology optimization of energy harvesting devices using piezoelectric materials," Structural and Multidisciplinary Optimization, vol. 38, no. 1, pp. 17-23, 2009.

[16] J. Y. Noh and G. H. Yoon, "Topology optimization of piezoelectric energy harvesting devices considering static and harmonic dynamic loads," Advances in Engineering Software, vol. 53, pp. 45-60, 2012.

[17] Z.-Q. Lin, H. C. Gea, and S.-T. Liu, "Design of piezoelectric energy harvesting devices subjected to broadband random vibrations by applying topology optimization," Acta Mechanica Sinica, vol. 27, no. 5, p. 730, 2011.

[18] G.-R. Liu and N. T. Trung, Smoothed finite element methods. CRC press, 2016.

[19] P. I. Kattan, MATLAB guide to finite elements: an interactive approach. Springer Science \& Business Media, 2010.

[20] T. Schlinquer, A. Mohand-Ousaid, and M. Rakotondrabe, "Displacement amplifier mechanism for piezoelectric actuators design using simp topology optimization approach," in IEEE Int Conference on Robotics and Automation. IEEE, 2018, pp. 1-7. 